\title{
Morphological and Immunohistochemical Features of the Pancreatic Duct System in Patients with Chronic Pancreatitis
}

\author{
Volodymyr G Yareshko, Anton V Yevseiev, Yurii A Mikheiev* \\ Department of Surgery and Minimally Invasive Technologies, Zaporizhia Medical Academy, Ukraine
}

*Corresponding author: Yurii A Mikheiev, PhD, Assistant Professor, Department of Surgery and Minimally Invasive Technologies, SI Zaporizhia Medical Academy of Post-Graduate Education Ministry of Health of Ukraine, Vintera boulevard 20, Zaporizhia, 69096, Ukraine

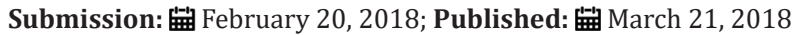

\begin{abstract}
Background: The duct cells make up to $30 \%$ of the human pancreas. One of the first signs of chronic pancreatitis is the abnormal increase in the number of duct brunches. The pancreatic ductal changes, periductal, inter- and intralobular fibrosis etc. are not the causes of chronic pancreatitis, but its consequences. The aim of the present study was to determine morphological and immunohistochemical features of the pancreatic duct system in patients with chronic pancreatitis, who underwent surgery for chronic pancreatitis.
\end{abstract}

Materials and methods: An immunohistochemical study of the surgical material of 40 patients with complicated chronic pancreatitis was carried out using antibodies to $\alpha$-SMA, desmin, fibronectin and type I collagen.

Results: A characteristic morphological picture of the chronic pancreatitis in development is a combination of fibrosis with deformation of the duct system due to periductal fibrosis with the expansion of interlobular fibrosis, thickening of intralobular connective tissue strands, as well as considerable crimp and widening of the ducts with retention cysts. Significant structural reorganization of the pancreatic duct system in patients with chronic pancreatitis was observed in $87.5 \%$ of cases, in $17.5 \%$ of cases the picture was supplemented by phenomena of acinar-ductal metaplasia with the transformation of acinar cells into ductal cells and the formation of small ductal-like structures, the so-called "tubular complexes". In 7.5\% of the cases in the main pancreatic duct, foci of atrophy of the epithelium were identified, which alternated with foci of papillary intraepithelial epithelial hyperplasia (PanIN). The development of pancreatic fibrosis in chronic pancreatitis is facilitated by the accumulation of $\alpha$-SMA+ activated pancreatic stellate cells synthesizing an excess of components of the extra-cellular matrix, in particular type I collagen. The mean area of type I collagen and the intensity of its expression were significantly lower with reduced $\alpha$-SMA expression $(\mathrm{p}<0.05)$, and significantly higher in cases of pronounced fibronectin expression $(\mathrm{p}<0.05)$.

Conclusion: The study of structural changes of the pancreatic duct system in patients with chronic pancreatitis using immunohistochemistry is one of the ways to understanding of pathogenesis and progressing of chronic pancreatitis.

Keywords: Chronic pancreatitis; Duct metaplasia; $\alpha$-SMA; Desmin; Fibronectin; Type I collagen

Aim

The aim of the study was to determine the morphological and immunohistochemical features of the ductal system of the pancreas of patients who had undergone resection and draining operation due to complications of chronic pancreatitis.

\section{Introduction}

Chronic pancreatitis is a disease of the pancreas in which recurrent inflammatory episodes result in replacement of pancreatic parenchyma by fibrous connective tissue. Chronic pancreatitis is characterized by specific changes in the structure and function of the pancreas, including atrophy of functional tissue, fibrosis and duct degeneration [1-3], a decrease in the number of islets of Langerhans [1]. Despite the large number of possible etiological factors, the main pathophysiological mechanisms of the development of chronic pancreatitis have much in common. Thus, with the preservation of secretory activity in conditions of obstruction of the ducts, their compensatory expansion and pancreatic secret infiltrates the surrounding tissue with the formation of edema of the pancreas, followed by intrapancreatic activation of digestive enzymes, in particular trypsinogen, which initiates proteolytic necrobiosis of pancreocytes [4]. In the future, this leads to the formation of "protein caps" pancreatic ducts, due to increased secretion of pancreatic proteins, which are the centers of calcification and lead to the formation of concernments in the ductal pancreatic system [5]. As a result, the epithelium of the ducts is affected, which forms molds and clogs of the ducts, thereby causing inflammatory changes and loss of functioning pancreatic parenchyma due to fibrosis $[4,6,7]$.

The duct cells make up to $30 \%$ of the mass of the human pancreas, compared to $1-2 \%$ of endocrine cells [8]. One of the first 
signs of HP is the abnormal increase in the number of brunch ducts. It has also been shown that in patients with HP in the ducts, the number of cells containing insulin increases, as well as cells that contain other endocrine and exocrine markers [9]. When modeling pancreatitis in a rat experiment was made, it was confirmed that cells of epithelial origin are involved in the regulation of the extracellular matrix (ECM) of the pancreas [10].

From the biochemical point of view, ECM consists of four main classes of macromolecules - collagens, proteoglycans, structural glycoproteins and elastin [11,12]. Structural strength and functional unity of ECM proteins play an important role in maintaining the organ-specific function of the pancreas $[12,13]$. Significant progress in understanding the development of fibrosis in chronic pancreatitis is associated with the identification of pancreatic stellate cells (PaSCs), which are capable of producing ECM, including excess [14].

For the first time lipid-containing cells were found in the human pancreas, rats and mice in 1982. Although the potential role of these cells in fibrogenesis was already discussed, another 8 years passed before scientists could isolate and characterize them [15]. PaSCs are located in the intralobular and intraacinar space of the human pancreas and have the characteristics of myofibroblasts: an examination of the $\alpha$-isoform of smooth muscle actin, the synthesis of collagen and fibronectin [16]. Also, PaSCs express intermediate filaments that are characteristic of myocytes - for example, desmin.

There are three basic mechanisms by which activation of PaSCs in chronic pancreatitis may occur: mechanical stress, production of cytokines and changes in ECM [14]. The content of fat droplets is inversely proportional to the expression of SMA and the synthesis of ECM. The transition from the latent "lipid-containing" form to "myofibroblastoid" with high proliferative properties is associated with the loss of drops of retinol, the development of endoplasmic reticulum and an increase in the synthesis of ECM proteins. Unlike the latent form, active or "synthesizing" is characterized by the synthesis of large volumes of collagen of the first type and fibronectin $[11,17]$.

The activation of the PaSCs follows their proliferation and migration. In cell culture, activated PaSCs rapidly proliferate and migrate under the control of chemotaxis toward the damaged tissue [18]. In addition to active migration to the injured sector, their absence in normal tissue is controlled by apoptosis. With the activation of PaSCs, a number of morphological changes occur, including changes to the myofibroblast-like phenotype, which synthesizes and secrets a large amount of type I collagen and fibronectin [19]. The shift in self-regulation of the PaSCs leads to an excessive accumulation of ECM and/or a reduced rate of its destruction, involving healthy functional-significant structures of the prostate in the process of fibrosis $[14,20]$.

\section{Materials and Methods}

Comprehensive histopathological and immunohistochemical study was performed in the histological and immunohistochemical laboratory of the University Hospital of Zaporozhye State Medical University based on postoperative material of 40 patients aged 28 to 74 years suffering from chronic pancreatitis, who were operated at the clinic of the department of surgery and minimally invasive technologies SI "Zaporozhye Medical Academy postgraduate education of the Ministry of Health of Ukraine".

For performing histological biopsies of pancreas were fixed in $10.0 \%$ neutral formalin, dehydrated in alcohols of increasing concentration and embedded in paraffin. Histological sections 3-5 $\mu \mathrm{m}$ thick were stained with hematoxylin and eosin, according to the Van Gyzon and Mallory-Trichrome methods. Immunohistochemical study was performed according to the standard procedure [21,22] using monoclonal mouse antibodies Alpha Smooth Muscle Actin against $\alpha$-isoform of smooth muscle actin ( $\alpha$-SMA) and desmin (Clone D33) against desmin (DAKO, USA), monoclonal rabbit antibodies Collagen I (Clone RAH C11) against type I collagen (Imtek, RF) and polyclonal rabbit antibodies Fibronectin against fibronectin (DAKO, USA). To do this, after dewaxing, high-temperature unmasking of antigens in Tris-EDTA buffer with pH 9 and blocking the activity of endogenous peroxidase in a $3 \%$ solution of hydrogen peroxide, incubation with primary antibodies was performed according to the recommendations of the manufacturer, followed by visualization of the product of immunohistochemical reaction using the EnVision detection system FLEX with diaminobenzidine (DAKO, USA).

Evaluation of immunohistochemical reaction with photodocumentation was performed using an Axioplan 2 microscope (Carl Zeiss, Germany) and a digital camera C5060WZ (Olympus, Japan). For photomorphometric study, 10 fields of vision were photographed in each case. To standardize the data obtained, the photos were taken under standardized conditions: an increase in the x200 microscope, a color (temperature) of 3200K, camera parameters F3.2 (aperture), 1/400 (shutter speed), ISO 100 (photosensitivity), manual white balance "one touch".

Quantitative determination of the levels of intensity and relative area of expression of the stromal markers was carried out using the ImageJ v.1.48 medical image processing software using the built-in Color Deconvolution plug-in and the $\mathrm{H}$ DAB color scheme (hematoxylin + DAB) to determine the area occupied by immunopositive structure in immunohistochemical preparations, and optical (densitometric) intensity of immunostaining according to the patented technique [23]. For the morphometric calculation of the quantitative content of the desired components, an automatically constructed histogram of the 8-bit half-tone image of the filtered DAB channel with software calculation of the optical density (Mean) and standard deviation (Std Dev) was used. For the subsequent morphometric measurement of the relative area in a halftone image, a standard threshold of sensitivity (the "Threshold" tool) was used to calculate the expression of $\alpha$-SMA and collagen I, and Reniy Entropy to calculate expression of desmin and fibronectin. Due to this procedure, the digital image was segmented, which provides the separation of all image pixels into two types - black and white. Then the percentage of the number of 
pixels of the digital image of the immunopositive reaction (black) was calculated to the total number of pixels in the image.

\section{Results and Discussion}

One of the important features associated with the course of $\mathrm{CP}$, is the order of development of characteristic microstructural changes in the pancreas. So, with the progression of fibrosis, the volume fraction of exocrine tissue decreases, the formation of fibrous tissue begins from the ducts, with subsequent attraction of perilobular space and penetration into the lobules [3]. In the pathohistological study of medicaments with a specific color of collagen (according to Van Gieson and Mallory-trichrome), fibrotic changes in all the patients with CP were characterized by a combination of pronounced perilobular fibrosis, which covered all lobules, widespread intralobular fibrosis (Figure 1A), as well as expressive periductal fibrosis near large, sharply defined, narrow and cystically dilated ducts. There was also a noticeable expansion of interlobular fibrous septum, a thickening of intralobular connective tissue strands directed deep into the lobules and connective tissue strands that surrounded the individual acini, as well as a significant crimp and widening of the ducts with retention cysts (Figure 1B).
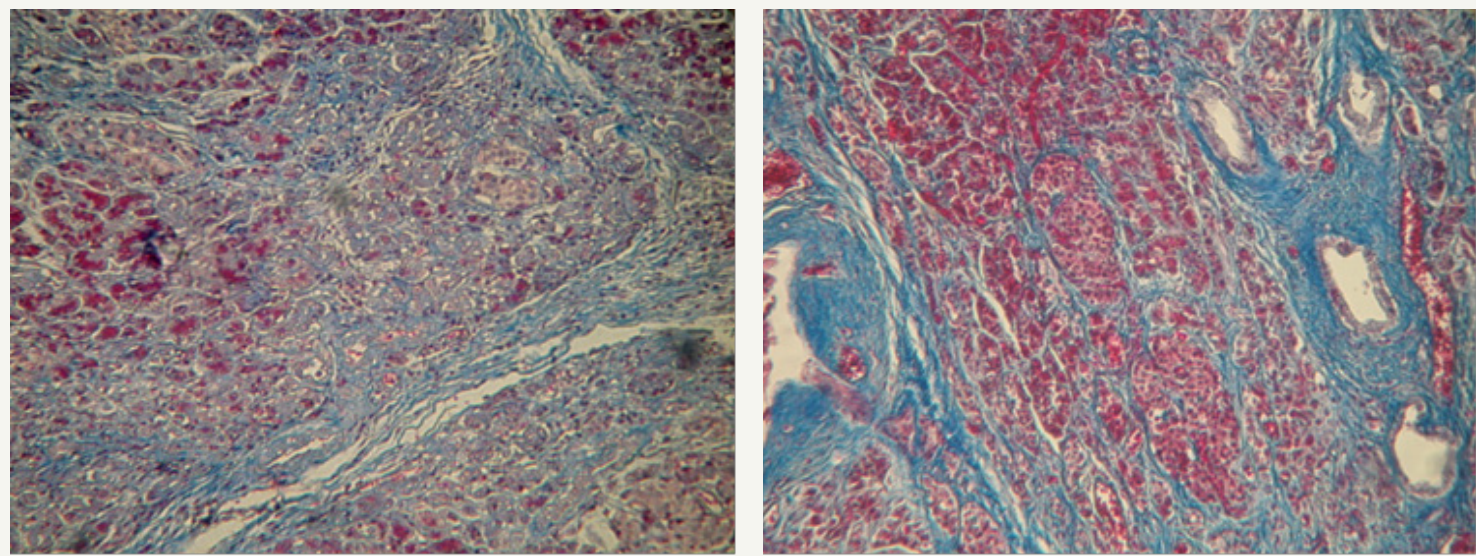

Figure 1: Pancreas with signs of fibrosis. Color Mallory-trichrome, $x 400$ : A) Perilobulary fibrosis, connective tissue strands penetrate into the pancreatic lobes, breaking the characteristic acinar structure; B) Intralobulary strands of connective tissue depth direction slices, tightly cover the expanded ducts.

In most cases, the deformation of the flow system was related to the number of the most notable structural changes. So, in $87.5 \%$ of cases near the unevenly narrowed and cystic-altered interlobular ducts noticeable concentric fibrosis was observed, protein plugs and concrements were observed in the holes of the ducts. In $7.5 \%$ of the cases in the main pancreatic duct, foci of atrophy of the protocol epithelium were identified, which alternated with foci of papillary intraepithelial epithelial hyperplasia; pancreatic intraepithelial neoplasia Pan IN.
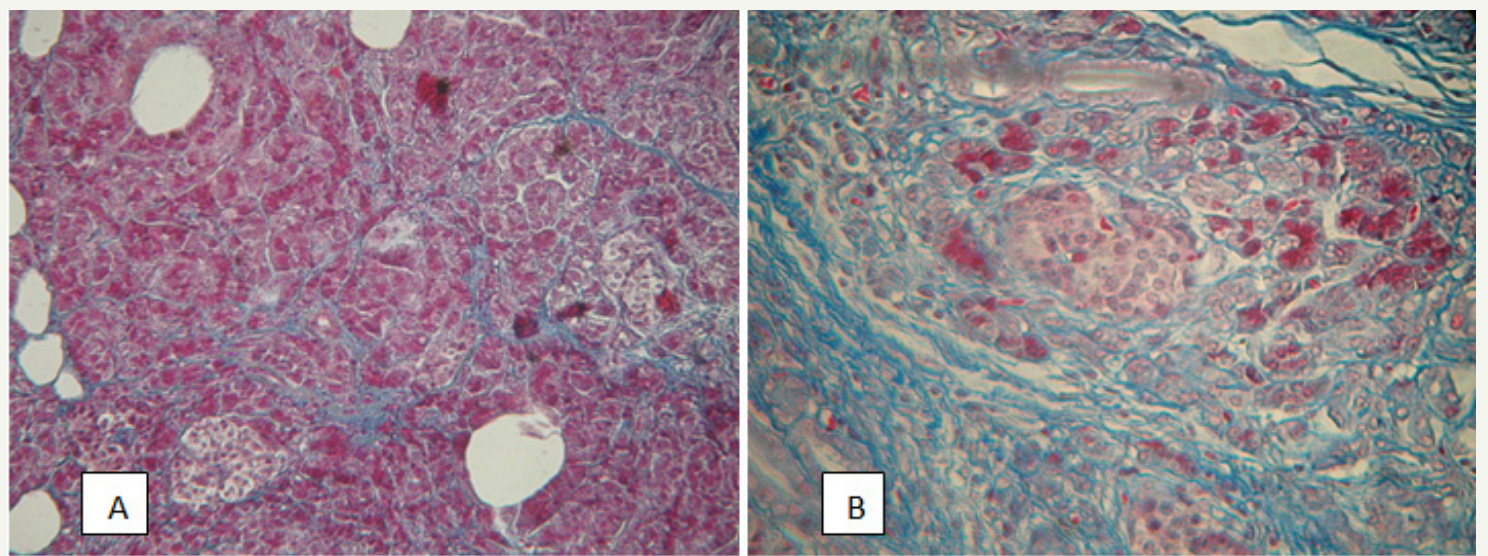

Figure 2: Pancreas with signs of fibrosis: A) Intralobular fibrosis, violation of the acinar structure, but with intact islets of Langerhans. Color Mallory-trichrome, uv. x200; B) On the site of the destroyed slices connective tissue penetrates into the islands. Color Mallory-trichrome, x400.

Among the acini, the islets of Langerhans were determined, both intact (Figure 2A) and tightly surrounded by fibrous tissue (Figure 2B). Recent research indicates that pancreatic islets begin to degrade only in the last stages of pancreatic fibrosis [23]. In our work, the involvement of the islets of Langerhans in the process of fibrosis was observed only in cases when the surrounding acini were almost completely replaced by a connective tissue.

The increase in the number of branch ducts during morphological examination was determined in the form of growth 
of ducts in two based types, which are characteristic for fibrosis of other parenchymal organs: continued, with the formation of a large number of parallel brunches of small and medium diameter (Figure
3A) and another, which differs by the deformation of the wall and the expansion of the section of the duct, with the formation of numerous branches of small diameter (Figure 3B).

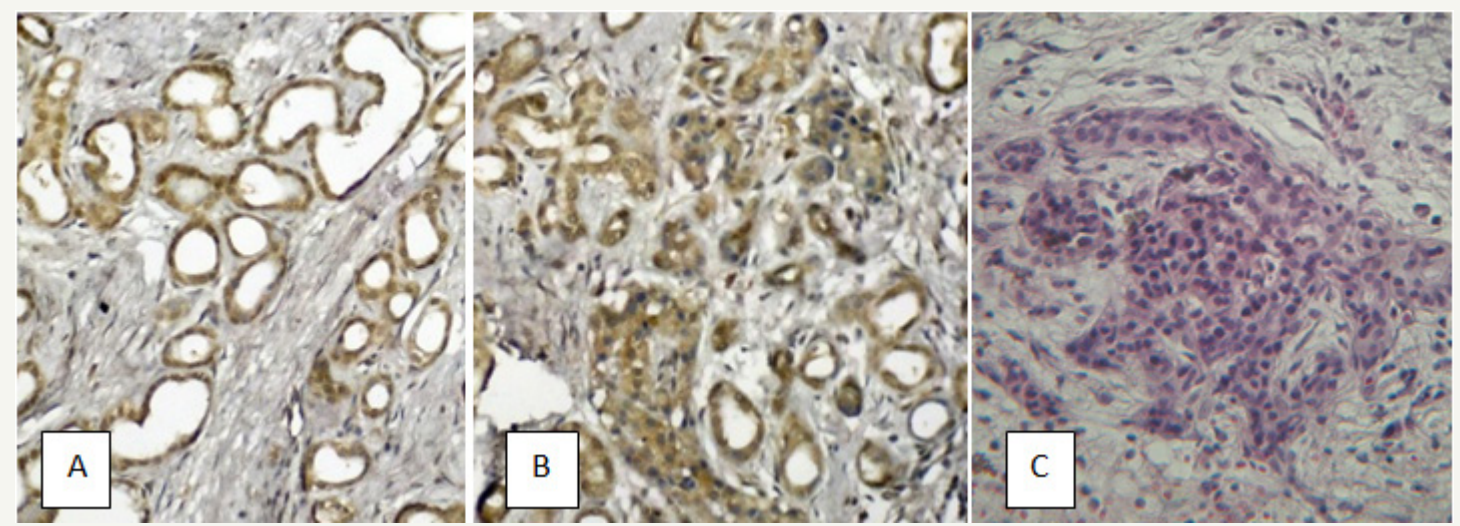

Figure 3: Pancreas with signs of fibrosis: A) Increased mass of ducts due to the extended type of growth. Immunohistochemical reaction with antibodies to fibronectin, x200; B) Increase in the mass of the ducts due to the offgrowth type of growth. Immunohistochemical reaction with antibodies to fibronectin, x200; C) The tubular complex. Staining with hematoxylin and eosin, $\mathrm{x} 400$.

The parenchyma of the pancreas, thus, was a small, atrophic acinus, surrounded by dense fibrous couplings, with an increased number of gaping ducts of different diameters. Also, in $17.5 \%$ of cases, phenomena of acinar-ductal metaplasia (Figure 3B) were observed. In the centers of the so-called acinar-ductal metaplasia, the transformation of some acinar cells into ductal cells was determined with the formation of small ductuloid-like structures, called "Tubular complexes," which according to the literature are the primary focus of regeneration of acinar tissue [3,23].
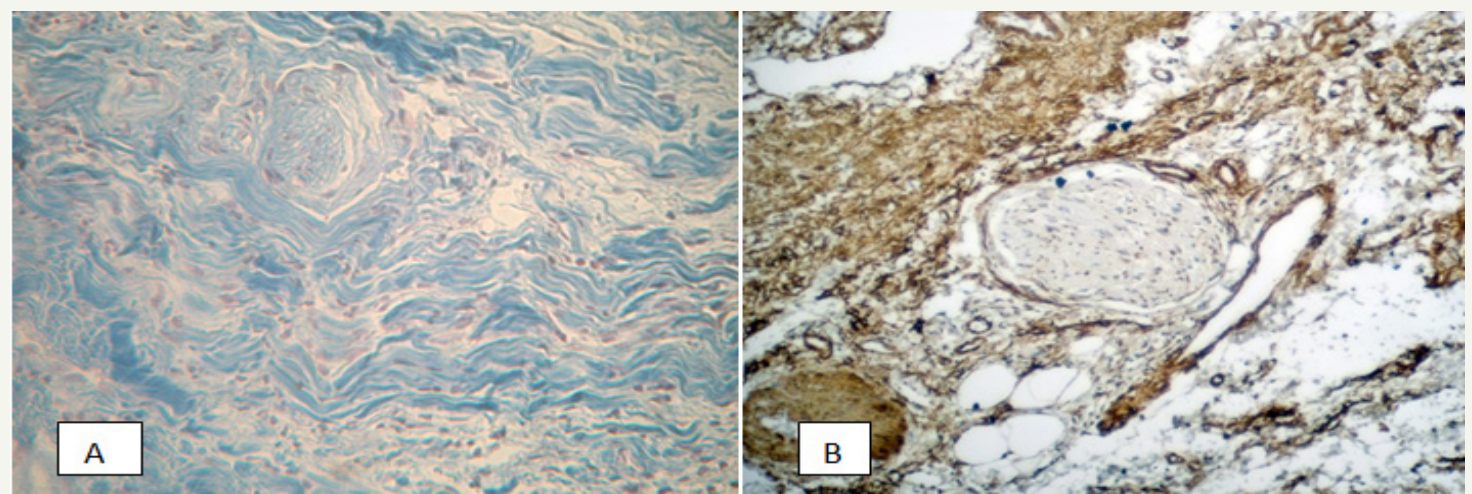

Figure 4: Perineural fibrosis of the pancreas in chronic pancreatitis. A) Color Mallory-trichrome, x200; B) Immunohistochemical reaction with antibodies $\mathrm{Rb}$ a-Hu Fibronectin, visualization system DAKO EnVision FLEX. x200.

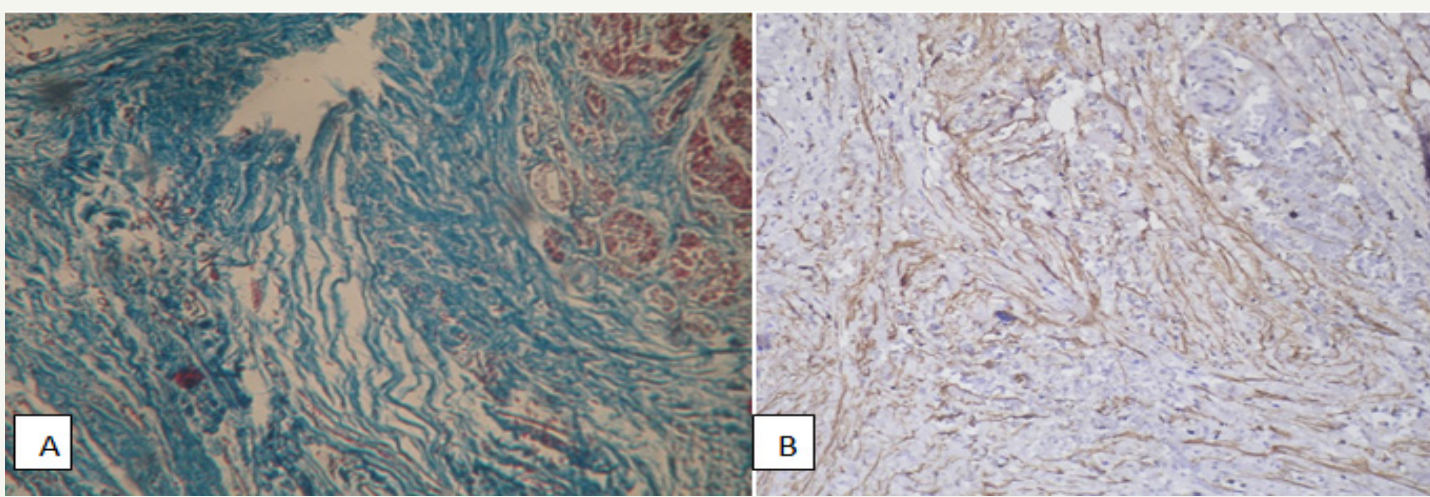

Figure 5: Expressed a-SMA-immunopositive staining of PaSCs and their processes, as well as the fibrillar component in the zone of pronounced pancreatic fibrosis. A) Color Mallory-trichrome, x200; B) Immunohistochemical reaction with antibodies Mo a-Hu Smooth Muscle Actin Alpha, visualization system DAKO EnVision FLEX. x200. 
In the fibrosis zones, a few focal immune cell infiltrates were identified, more characteristic of the acute phase of the disease. In $45 \%$ of cases, the examined preparations showed the presence of perineural fibrosis (Figure 4), which potentially aggravates the pain reaction in chronic pancreatitis. Immunohistochemical and morphometric studies that were conducted have shown that in areas of severe fibrosis in chronic pancreatitis, a number of cellular and fibrillar markers is expressed. The results are shown in Table 1.

Table 1: Results of photocytometric morphometry of immunohistochemical preparations, $\mathrm{M} \pm \sigma$.

\begin{tabular}{|c|c|c|c|c|c|c|c|c|}
\hline & \multicolumn{2}{|c|}{$\alpha$-SMA } & \multicolumn{2}{|c|}{ Desmin } & \multicolumn{2}{|c|}{ Fibronectin } & \multicolumn{2}{|c|}{ Collagen I } \\
\hline & Sотн & Mean & Sотн & Mean & Sотн & Mean & Sотн & Mean \\
\hline$M$ & $17,71 \%$ & 44,93 & $1,09 \%$ & 4,40 & $12,31 \%$ & 34,20 & $26,47 \%$ & 89,90 \\
\hline$\sigma$ & $1,65 \%$ & 4,66 & $0,08 \%$ & 0,63 & $1,53 \%$ & 4,48 & $3,01 \%$ & 5,20 \\
\hline
\end{tabular}

In particular, in the fibrosis zones a large number of activated $\alpha$-SMA + pancreatic stellate cells (PaSCs) is detected. The pattern of expression of this marker is characterized by marked $\alpha$-SMAimmunopositive staining as a PSC and their processes, and partly - fibrillar component (Figure 5).

Primary statistical processing of morphometry data revealed a direct relationship between the expression of $\alpha$-SMA and the area of type I collagen, but this relationship was not significant $(r=+0.321$, $\mathrm{p}<0,05$ ). With the aim of further objectifying statistical studies, we isolated a group of cases characterized by low $\alpha$-SMA expression $(\mathrm{n}=9)$. A further study using medical statistics showed that the average area of type I collagen and the intensity of its expression in this group was significantly lower than in the rest of the biopsies $(\mathrm{p}<0,05)$.

In contrast to inactive PaSCs localized around the pancreatic lobes and in the outer layer of the main pancreatic duct wall, activated $\alpha$-SMA-positive PaSCs play an important role in the development of fibrosis in chronic pancreatitis [1,3], secreting in addition to collagen a large number of other ECM components, among which an important role is played by fibronectin (extracellular matrix protein) and desmin (intermediate filament protein). In our study, fibronectin was visualized in the connective tissue, not affecting the

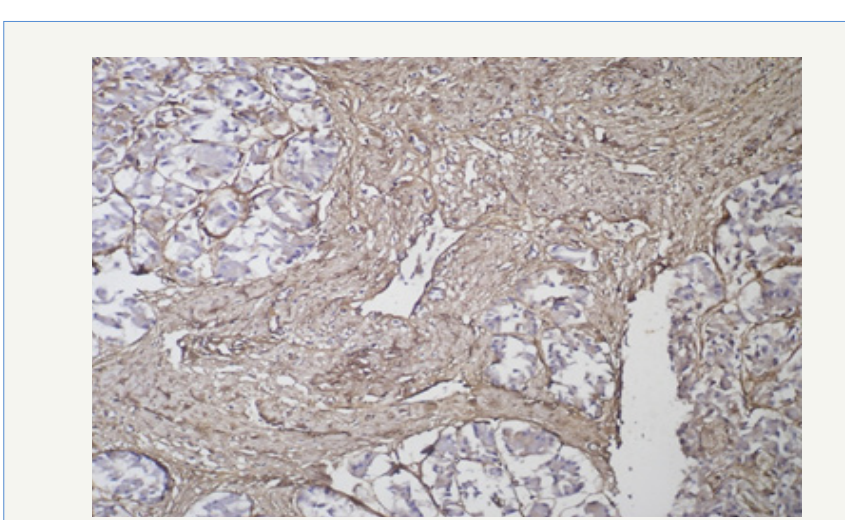

Figure 6: Expression of fibronectin in the connective tissue surrounding the panreatic acinus. Immunohistochemical reaction, visualization system DAKO Envision FLEX. x200.

acini, islets and epithelium of large ducts (Figure 6).

In contrast, desmin was defined as in connective tissue, which was characterized by a lower intensity of immunostaining, and in acinar tissue and in the epithelial tissue of the pancreatic ducts (Figure 7). The pattern of its expression in zones of fibrosis in chronic pancreatitis was similar to that of $\alpha$-SMA, but in $27,5 \%$ of cases the intensity of staining was below the threshold for determination by morphometric methods.
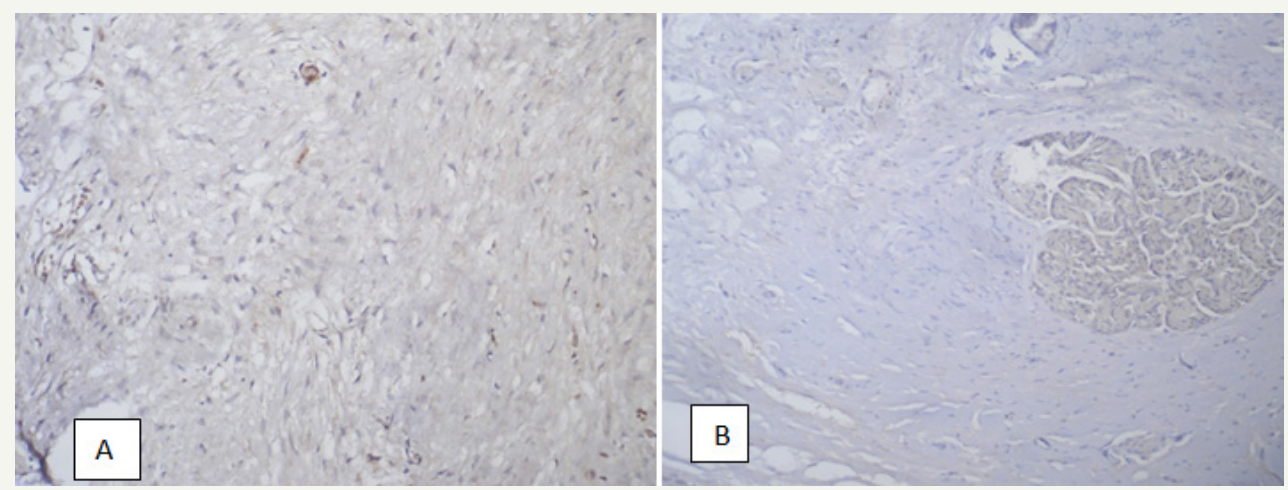

Figure 7: Expression of desmin in the connective (A), and in the acinar tissue of the pancreas (B). Immunohistochemical reaction, visualization system DAKO EnVision FLEX. x200.

Fibronectin, which is synthesized by virtually all cell types and which is presented in the pancreas in insoluble form as a fibrillar network on the cell surface and in the extracellular matrix [12], binds to aggregating procollagen fibrils and changes the kinetics of fibril formation in the pericellular matrix. We isolated a group of cases characterized by high expression of fibronectin $(n=9)$. A further study using medical statistics showed that the average area of type I collagen and the intensity of its expression in this group was significantly higher than in the rest of the biopsies $(\mathrm{p}<0,05)$.

Thus, in the immunohistochemical study of the material of patients with chronic pancreatitis, it was found that a large number 
of activated PaSCs expressing $\alpha$-SMA and desmin are concentrated in the zones of pancreatic fibrosis. Also in these zones, a significant accumulation of fibronectin was found, which is synthesized in excess by activated PaSCs. The pattern of immunohistochemical staining of type I collagen in the zones of severe pancreatic fibrosis is characterized by a low and moderate intensity with a moderate level of its expression.

Conducted immunohistochemical studies have shown that the presence of a significant number of $\alpha$-SMA-positive PaSCs, as well as a significant amount of fibronectin indicate the activity of the fibrous process. The obtained data of morphological changes in pancreatic tissue in the study of surgical material in patients with complications of chronic pancreatitis are consistent with the latest data obtained in the study of fibrosing processes in the prostate and other parenchymatous organs $[10,16,19]$. The revealed perineural infiltration and perineural fibrosis in the parenchyma of the pancreas once again confirm the data about the complex mechanism of pain syndrome in this group of patients, which can not be explained only by central ductal pancreatic hypertension.

The process of activation of PaSCs is associated with structural disturbances of the duct system and destruction of the acini $[3,23]$, which is consistent with the data of our study, where a pronounced pattern of involvement of the duct system in the process of fibrosis was observed in $87.5 \%$ of cases. In addition, the centers of pancreatic intraepithelial neoplasia Pan IN in patients with chronic pancreatitis, once again show that chronic pancreatitis is an optional precancerous condition. In this case, neoplasia begins with the epithelium, more often the main pancreatic duct, which can subsequently lead to the development of ductal pancreatic adenocarcinoma. This fact justifies the performance of virsuncectomy in the surgical treatment of chronic pancreatitis.

\section{Conclusion}

I. A characteristic morphological picture of the development of chronic pancreatitis is the deformation of the duct system against the background of fibrosis with considerable crimp and dilatation of the ducts due to periductal fibrosis with the expansion of interlobular fibrosis septum, thickening of intralobular connective tissue strands and retention cysts.

II. The pronounced structural rearrangement of the pancreatic duct system in patients with chronic pancreatitis was observed in $87.5 \%$ of cases, in $17.5 \%$ of cases the picture was supplemented by phenomena of acinar-ductal metaplasia with the transformation of acinar cells into ducts and the formation of small duktuloid-like structures, called "Tubular complexes". In $7.5 \%$ of the cases in the main pancreatic duct, foci of atrophy of the protocol epithelium were identified, which alternated with foci of papillary intraepithelial epithelial hyperplasia (PanIN).

III. The development of pancreatic fibrosis in chronic pancreatitis causes due to the accumulation of $\alpha$ - SMA + activated pancreatic stellate cells synthesizing excess of extra components of the cellular matrix, especially collagen I type. The mean area of type I collagen and the intensity of its expression were significantly lower with reduced $\alpha$-SMA expression $(\mathrm{p}<0,05)$, and significantly higher in cases of pronounced fibronectin expression $(\mathrm{p}<0,05)$.

\section{Competing Interest}

The authors declare that he has no competing interests.

\section{References}

1. Lew D, Afghani E, Pandol S (2017) Chronic Pancreatitis: Current Status and Challenges for Prevention and Treatment. Dig Dis Sci 62(7): 17021712.

2. Yadav D, Lowenfels A (2013) The Epidemiology of Pancreatitis and Pancreatic Cancer. Gastroenterology 144(6): 1252-1261.

3. Manohar M, Verma A, Venkateshaiah S, Sanders N, Mishra A (2017) Pathogenic mechanisms of pancreatitis. World J Gastrointest Pharmacol Ther 8(1): 10-25.

4. Mayev IV, Kazyulin AN, Kucheryavy Yu A (2005) Chronic pansreatitis. Moskow: medicine $240 \mathrm{p}$

5. Bynigeri R, Jakkampudi A, Jangala R, Subramanyam C, Sasikala M, et al. (2017) Pancreatic stellate cell: Pandora's box for pancreatic disease biology. World J Gastroenterol 23(3): 382-405.

6. Strobel O, Dadabaeva N, Felix K, Hackert T, Giese N, et al. (2015) Isolation and culture of primary human pancreatic stellate cells that reflect the context of their tissue of origin. Langenbecks Arch Surg 401(1): 89-97.

7. Haqq J, Howells LM, Garcea G, Metcalfe MS, Steward WP, et al. (2014) Pancreatic stellate cells and pancreas cancer: current perspectives and future strategies. Eur J Cancer 50(15): 2570-2582.

8. Kawase T, Yasui Y, Nishina S, Hara Y, Yanatori I, et al. (2015) Fibroblast activation protein- $\alpha$-expressing fibroblasts promote the progression of pancreatic ductal adenocarcinoma. BMC Gastroenterol 15: 109.

9. Komar H, Serpa G, Kerscher C, Schwoegl E, Mace T, et al. (2017) Inhibition of Jak/STAT signaling reduces the activation of pancreatic stellate cells in vitro and limits caerulein-induced chronic pancreatitis in vivo. Sci Rep 7(1): 1787.

10. Sakaguchi Y, Inaba M, Kusafuka K, Okazaki K, Ikehara S (2006) Establishment of Animal Models for Three Types of Pancreatitis and Analyses of Regeneration Mechanisms. Pancreas 33(4): 371-381.

11. Bolm L, Cigolla S, Wittel UA, Hopt UT, Keck T, et al. (2017) The Role of Fibroblasts in Pancreatic Cancer: Extracellular Matrix Versus Paracrine Factors. Transl Oncol 10(4): 578-588.

12. Gress T, Menke A, Bachem M, Müller-Pillasch F, Ellenrieder V, et al. (1998) Role of Extracellular Matrix in Pancreatic Diseases. Digestion 59(6): 625-637.

13. Patel M, Fine DR (2005) Fibrogenesis in the pancreas after acinar cell injury. Scandinavian Journal of Surgery 94: 108-111.

14. Apte M, Pirola RC, Wilson JS (2015) Pancreatic stellate cell: physiologic role, role in fibrosis and cancer. Cur Opin Gastroenterol 31(5): 416-423.

15. Bachem MG, Schneider E, Gross H, Weidenbach H, Schmid RM, et al. (1998) Identification, culture, and characterization of pancreatic stellate cells in rats and humans. Gastroenterology 115(2): 421-432.

16. Allam A, Thomsen AR, Gothwal M, Saha D, Maurer J, et al. (2017) Pancreatic stellate cells in pancreatic cancer: In focus. Pancreatology 17(4): 514-522.

17. Sirenko O Yu (2010) Pancreatic stellate cells as a morphological basis for the development of pancreatic fibrosis. Morphology 4(1): 5-12.

18. Durko L, Wlodarski W, Stasikowska-KO, Wagrowska-DM, Danilewicz M, et al. (2017) Expression and Clinical Significance of Cancer Stem Cell 
Markers CD24, CD44, and CD133 in Pancreatic Ductal Adenocarcinoma and Chronic Pancreatitis. Disease Markers 17: 3276806.

19. Apte MV, Pirola RC, Wilson JS (2012) Pancreatic stellate cells: a starring role in normal and diseased pancreas. Front Physiol 3: 344.

20. von Ahrens D, Bhagat TD, Nagrath D, Maitra A, Verma A (2017) The role of stromal cancer-associated fibroblasts in pancreatic cancer. J Hematol Oncol 10(1): 76.
21. Dabbs DJ (2010) Diagnostic immunohistochemistry. (3 ${ }^{\text {rd }}$ edn), Ch. Livingstone, New York, USA, p. 941.

22. (2015) Methodfor photochemicalmorphometry ofimmunohistochemical slides. Ukraine.

23. Oshmyanska NY (2014) Death and regeneration of the pancreatic islets in relation to the development of fibrosis in patients with chronic pancreatitis. Gastroenterology 4(54): 88-95.

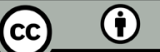

Creative Commons Attribution 4.0 International License

For possible submissions Click Here

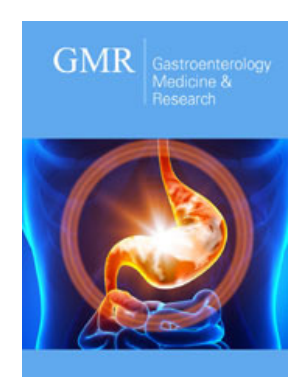

\section{Gastroenterology Medicine \& Research}

\section{Benefits of Publishing with us}

- High-level peer review and editorial services

- Freely accessible online immediately upon publication

- Authors retain the copyright to their work

- Licensing it under a Creative Commons license

- Visibility through different online platforms 